\title{
Replacement of dichloromethane within chromatographic purification: a guide to alternative solvents $\dagger+$
}

\author{
Donna S. MacMillan, ${ }^{a}$ Jane Murray, ${ }^{b}$ Helen F. Sneddon, ${ }^{c}$ Craig Jamieson ${ }^{a}$ and Allan J. B. Watson ${ }^{* a}$
}

Received 30th August 2012, Accepted 28th September 2012

DOI: 10.1039/c2gc36378j

Replacement of dichloromethane as the bulk medium within chromatographic purification has been evaluated with a broad range of molecules containing functionality common within Medicinal Chemistry programmes. Analysis of the data set has generated a set of general guidelines to assist in the selection of alternative solvents for $\mathrm{CH}_{2} \mathrm{Cl}_{2}$ as the bulk media in these ubiquitously employed processes.

Replacement of hazardous solvents in order to achieve greater sustainability and/or to reduce both environmental and operational costs is a key emerging consideration within the pharmaceutical industry. ${ }^{1}$ A number of reports have recently emerged from leading pharmaceutical companies detailing the drivers and requirements for increasing the sustainability of their overall processes through adoption of green chemistry principles and, indeed, have signposted a change in solvent selection as a primary method of achieving this. ${ }^{1-3}$ In particular, chlorinated solvents such as $\mathrm{CH}_{2} \mathrm{Cl}_{2}$ and $\mathrm{CHCl}_{3}$ are two common solvents of routine and widespread use that possess significant hazardous toxicity to both humans and the environment and which require more careful and costly disposal. ${ }^{4}$ Consequently, there is a strong desire to supplant these systems with more benign alternatives.

In relation to this, purification is by far the largest consumer of solvent within any synthetically aligned research programme. Indeed in the context of pharmaceutical research, on average solvent has been estimated to constitute some $56 \%$ of the total material used to manufacture active pharmaceutical ingredients. $^{2 a}$ Accordingly, moving to a more environmentally conservative solvent selection within chromatographic purification could therefore be expected to have the largest single impact on the overall sustainability of chemical synthesis endeavours.

\footnotetext{
${ }^{a}$ Department of Pure and Applied Chemistry, WestCHEM, University of Strathclyde, Thomas Graham Building, 295 Cathedral Street, Glasgow, G11XL,UK.E-mail: allan.watson.100@strath.ac.uk;

Fax: + 44 (0)141 548 4822; Tel: +44 (0)141548 2439

${ }^{b}$ Sigma-Aldrich, The Old Brickyard, New Road, Gillingham, Dorset, SP8 4XT, UK

${ }^{c}$ Green Chemistry Performance Unit, GlaxoSmithKline, Medicines

Research Centre, Gunnels Wood Road, Stevenage, Hertfordshire,

SG1 2NY, UK

$\dagger$ Dedicated to Professor William J. Kerr on the occasion of his 50th birthday.

Electronic supplementary information (ESI) available: Calculated molecular properties of all compounds, graphs for $R_{\mathrm{f}} v s$. \% modifier for each bulk solvent, box plot analyses, and Spotfire analyses. See DOI:
} $10.1039 / \mathrm{c} 2 \mathrm{gc} 36378 \mathrm{j}$
However, while this may seem reasonably straightforward in principle, the lack of solid data in this area presents a barrier to the general adoption of these more environmentally acceptable approaches.

Equielutropic series have been constructed for a range of conventional solvents, ${ }^{5}$ however, these are heavily skewed towards solvents with major regulatory and/or toxicological issues, for example chlorinated, toluene, hexane. This is presumably due to sustainability not being a primary concern during the time period in which this analysis was conducted (in the 1960s). As such, little equivalent data exists for alternative solvents with fewer issues that can be employed as replacements.

Having said this, a very recent contribution in this area from Amgen has endeavoured to provide a guide to alternative solvent selection for replacement of $\mathrm{CH}_{2} \mathrm{Cl}_{2}$ in purification. ${ }^{3}$ This excellent study has focused on the use of alcohol-( $\mathrm{MeOH}, \mathrm{EtOH}$, $\mathrm{i}-\mathrm{PrOH})$ and additive-modified $\left(\mathrm{AcOH}, \mathrm{NH}_{4} \mathrm{OH}\right)$ mixtures of heptanes, EtOAc, and tert-butyl methyl ether (TBME) for the purification of a range of 26 drug-like molecules and has presented a modern equielutropic series based on these mixtures in comparison to $\mathrm{MeOH}-\mathrm{CH}_{2} \mathrm{Cl}_{2}$.

In a complementary approach, we recently sought to generate specific and comprehensive data to assist in the selection of alternative solvents as replacements for $\mathrm{CH}_{2} \mathrm{Cl}_{2}$ within chromatographic purification using binary eluent systems. The current study will greatly assist in the selection of greener alternative eluents and we believe will help facilitate widespread adoption of more sustainable eluents in chromatographic purification processes.

\section{Results and discussion}

\section{Methods}

Our approach towards identifying potential replacements for chlorinated solvents, particularly $\mathrm{CH}_{2} \mathrm{Cl}_{2}$, in purification was based on thin layer chromatography (TLC) analysis. This was similar to the approach taken by Neher in 1964 (the approach that led to the original equielutropic series $)^{5}$ and to that recently adopted by Amgen. ${ }^{3}$ To ensure the greatest opportunity for adoption of any developed method, we focused primarily on binary eluent mixtures comprising of a bulk solvent modified by $\mathrm{MeOH}$ (or i-PrOH, vide infra) as we believed that these would be more convenient to the practicing chemist. Each compound from our selected library would be analysed by TLC at various 


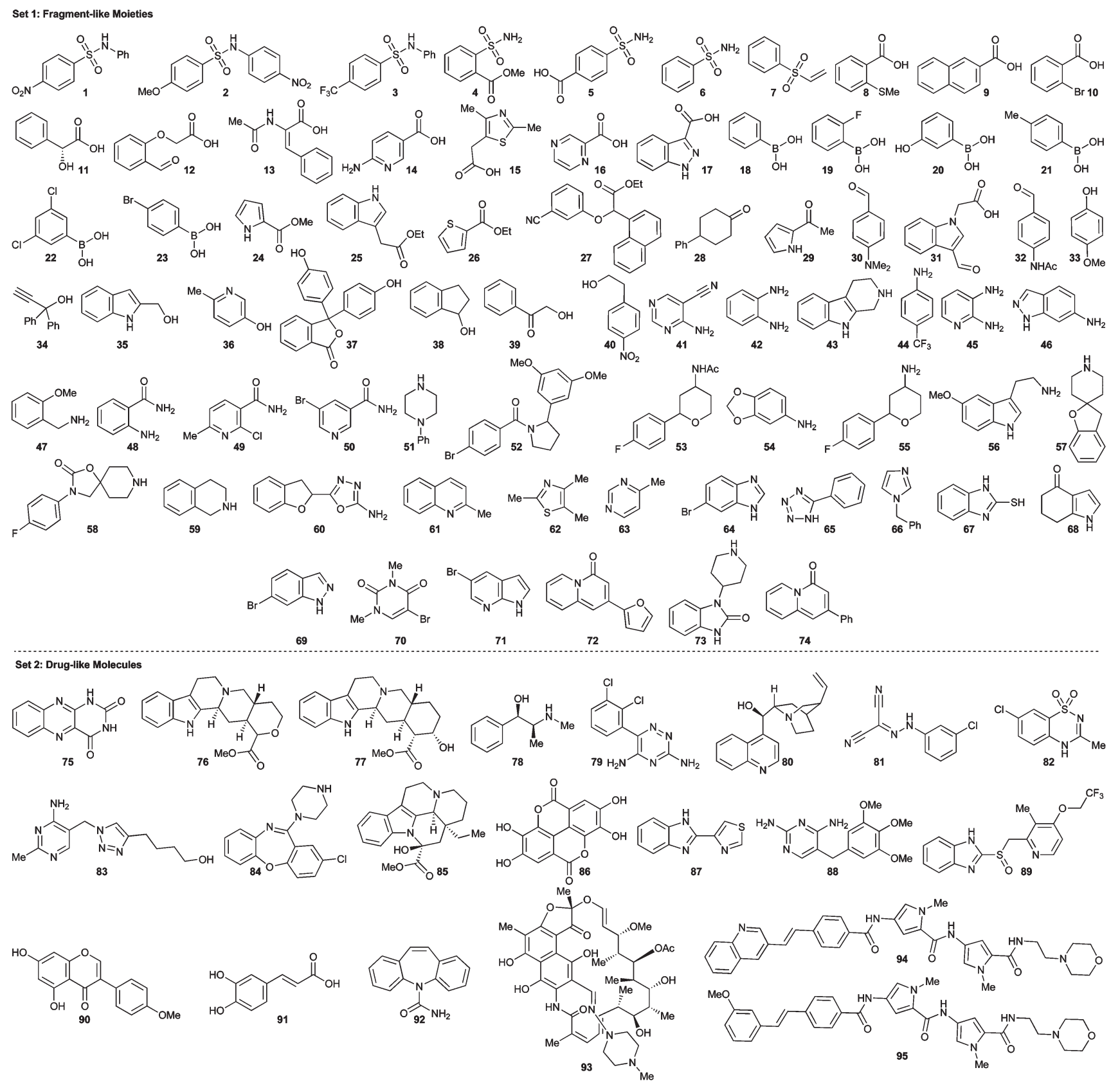

Fig. 1 Compound library evaluated.\$

compositions of modifier: bulk medium with analyses performed in triplicate to ensure reproducibility.

To initiate our study, we first selected a range of small leadlike molecules that displayed broad coverage of the functional group landscape. In addition to this and in attempts to ensure our data was as applicable and relevant as possible, we also selected a range of structurally more complex molecules, several of which were previously marketed drug molecules and others which had been prepared as part of on-going academic medicinal chemistry projects. ${ }^{6}$ In total, we surveyed 95 compounds -74 fragment-like moieties and 21 larger, drug-related molecules (sets 1 and 2, respectively, Fig. 1).
To ensure our data set was as realistic and as representative as possible, we performed principal component analysis (PCA) of the 95 compounds based on six descriptors: ${ }^{7}$ (i) molecular weight (94-822); (ii) number of hydrogen bond donors (0-6); (iii) number of hydrogen bond acceptors (0-12); (iv) number of rotatable bonds $(0-14)$; (v) X $\log P(-1.7$ to 3.2$)$; and (vi) polar surface area $\left(12-220 \AA^{2}\right)$. A scatter diagram of this analysis (Fig. 2) clearly suggests diverse coverage of the chemical landscape: the $x$-axis (PCA dimension 0 ) is mainly a function of molecular weight and polar descriptors while the $y$-axis (PCA dimension 1) is mainly a lipophilicity descriptor. Inspection of the data suggests that there is a slight bias for the lower 


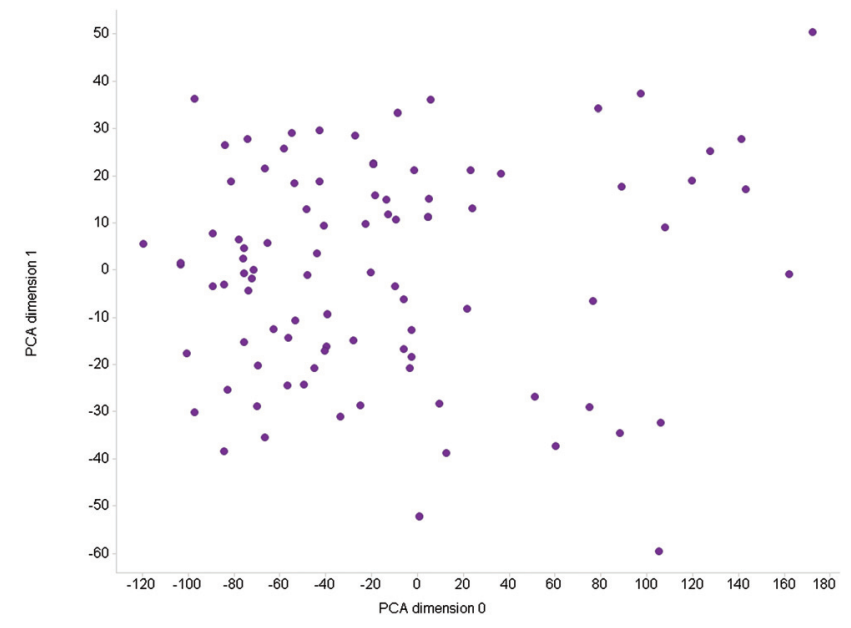

Fig. 2 PCA of the 95-substrate set.

molecular weight side of the scatter graph. This is likely to be a consequence of the majority of low molecular weight compounds (63 compounds in the molecular weight range 94-221), although, having stated this, there is still an excellent distribution of molecular properties within the data set and these compounds are more likely to be of relevance within a pharmaceutical research and development setting.

In terms of the alternative bulk media, we decided to employ several emerging or existing solvents to use in conjunction with the modifier. Specifically, we evaluated cyclopentyl methyl ether (CPME) ${ }^{8}$ TBME, 2-methyl tetrahydrofuran (2-MeTHF), dimethyl carbonate (DMC), and EtOAc. For the modifier, with the exception of EtOAc, we elected to use $\mathrm{MeOH}$ as it is one of the most widely used modifiers for polar compound chromatography and would enable a direct comparison with $\mathrm{MeOH}-$ $\mathrm{CH}_{2} \mathrm{Cl}_{2}$ mixtures. For EtOAc, i-PrOH was employed as the modifier based on emerging and promising data for this binary eluent. ${ }^{9}$ In addition, the Amgen group assessed $\mathrm{MeOH}-\mathrm{EtOAc}$ mixtures in their recent study and had compared this to $\mathrm{MeOH}-$ $\mathrm{CH}_{2} \mathrm{Cl}_{2}{ }^{3}$ Overall, this would provide five different binary eluent systems to compare with $\mathrm{MeOH}-\mathrm{CH}_{2} \mathrm{Cl}_{2}$ mixtures.

\section{Analysis}

A representative combined chart with $R_{\mathrm{f}}$ plotted against \% modifier for each of the six solvent systems for compound $\mathbf{8 9}$ is shown in Fig. 3. Equivalent data was generated for all 95 compounds with the associated error in average $R_{\mathrm{f}}$ values remaining consistently very low. We also observed reliably excellent linear correlation throughout the data sets, with $R^{2}$ values of $>0.95$.

With the data in hand, we sought to establish trends with which to form the basis for guidelines to direct use of these alternative eluent systems. A useful analysis of the data set was achieved through a simple box plot. Fig. 4 shows a box plot of $R_{\mathrm{f}}$ (y-axis) vs. $10 \%$ modifier in each of the bulk media (Table 1 displays the $R_{\mathrm{f}}$ ranges and medians for each eluent). We also conducted the same box plot analysis for the 5\% and $20 \%$ modifier levels and achieved similar distributions. This analysis gives a usefully condensed assessment of the $R_{\mathrm{f}}$ data for each of the eluent mixtures. The relative distribution of the data for the 95 compounds and for each of the binary mixtures can be clearly

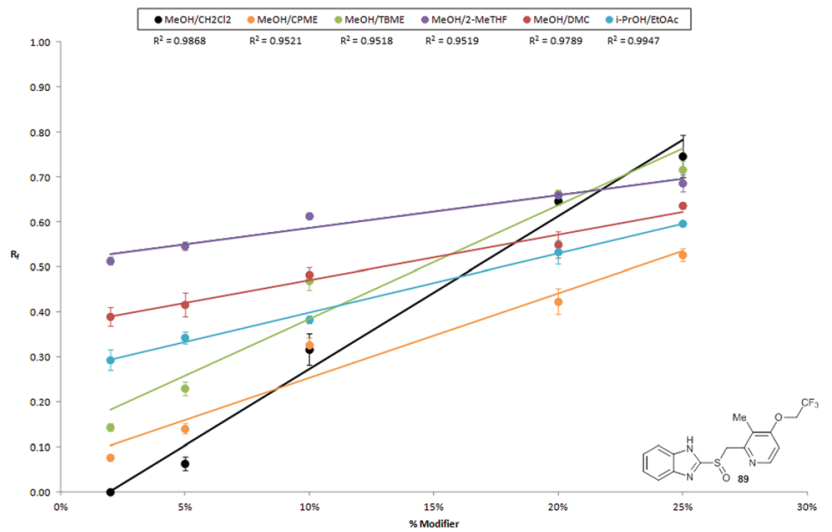

Fig. 3 Illustrative graph of eluent evaluation.

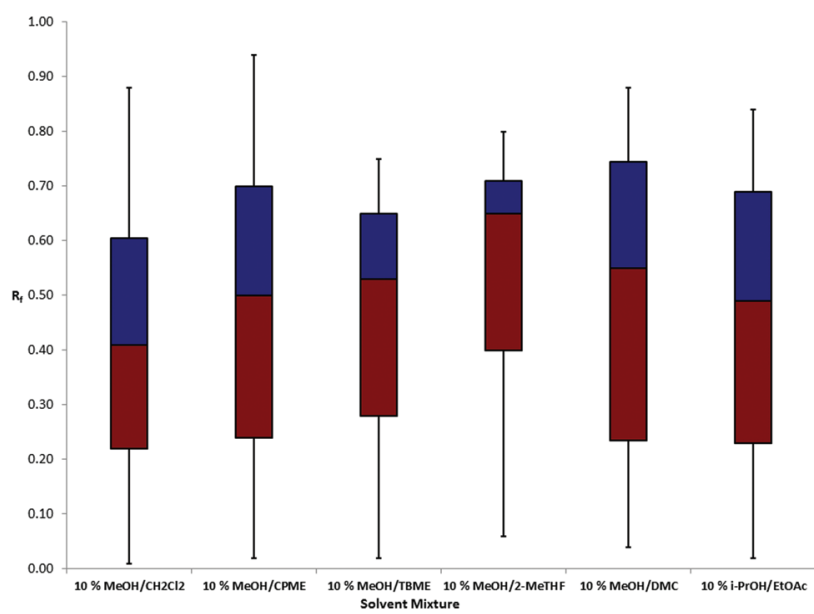

Fig. 4 Box plot analysis of $R_{\mathrm{f}} v s$. eluent at $10 \%$ modifier level.t.

Table $1 R_{\mathrm{f}}$ ranges and medians for box plot analysis in Fig. $4^{a}+$

\begin{tabular}{llll}
\hline Entry & Eluent & $R_{\mathrm{f}}$ range & Median \\
\hline 1 & $\mathrm{MeOH}-\mathrm{CH}_{2} \mathrm{Cl}_{2}$ & $0.01-0.88$ & 0.41 \\
2 & $\mathrm{MeOH}-\mathrm{CPME}$ & $0.02-0.94$ & 0.50 \\
3 & $\mathrm{MeOH}-\mathrm{TBME}$ & $0.02-0.75$ & 0.53 \\
4 & $\mathrm{MeOH}-2-\mathrm{MeTHF}$ & $0.06-0.80$ & 0.65 \\
5 & $\mathrm{MeOH}-\mathrm{DMC}$ & $0.04-0.88$ & 0.55 \\
6 & i-PrOH-EtOAc & $0.02-0.84$ & 0.49 \\
${ }^{a} 10 \%$ modifier in bulk solvent. & & \\
\hline
\end{tabular}

seen: each of the four quadrants indicates that $25 \%$ of the compounds can be found within that particular $R_{\mathrm{f}}$ range.

Firstly, the data for $\mathrm{MeOH}-\mathrm{CH}_{2} \mathrm{Cl}_{2}$ was spread reasonably well and with relatively good quadrant consistency over the $R_{\mathrm{f}}$ range: each of the data quadrants was of comparable magnitude and the data range covers the $R_{\mathrm{f}}$ range $0.01-88$, although there was a noted slight bias for the lower end of the $R_{\mathrm{f}}$ range (median $\left.R_{\mathrm{f}}=0.41\right)$. Comparing the data for the other five alternative eluent systems, the following observations can be made: (i) The data for the CPME-based system was excellent and, in fact, this analysis suggests that the CPME data is actually superior to the $\mathrm{CH}_{2} \mathrm{Cl}_{2}$ system, as the quadrants were more homogenous (median $=R_{\mathrm{f}} 0.50$ ) and the dynamic $R_{\mathrm{f}}$ range was wider 


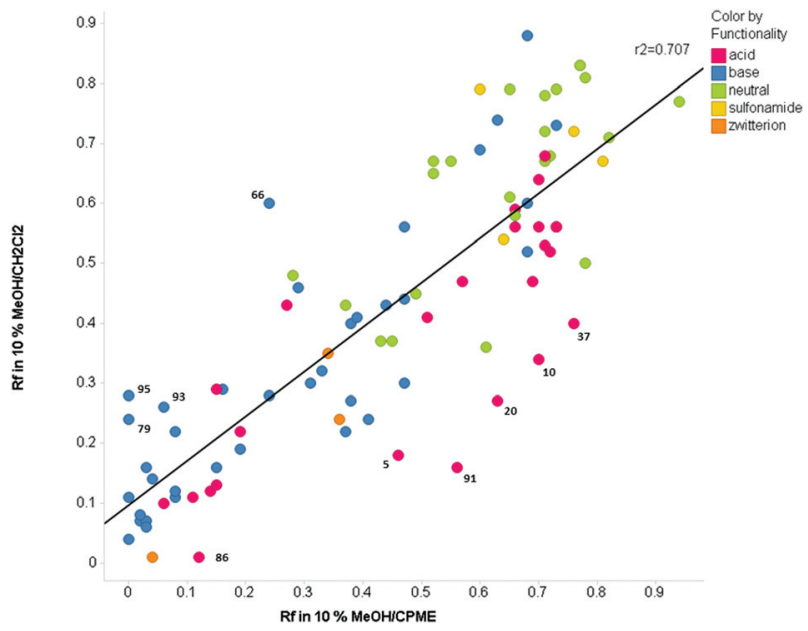

Fig. 5 Spotfire analysis of $\mathrm{MeOH}-\mathrm{CH}_{2} \mathrm{Cl}_{2}$ data $v$. $\mathrm{MeOH}-\mathrm{CPME}$ data at $10 \%$ modifier.

(0.02-0.94). This may translate to an improved achievable chromatographic resolution with the CPME-based eluent when employed in a purification scenario. (ii) Both the TBME- and 2-MeTHF-based eluents have comparatively greater compression in their data sets. For example, for 2-MeTHF, 50\% of the total data is found in the $0.65-0.80 R_{\mathrm{f}}$ range. This truncation of the higher $R_{\mathrm{f}}$ quadrants (median $R_{\mathrm{f}}=0.53$ and 0.65 , respectively) suggests a lower utility for replacing $\mathrm{CH}_{2} \mathrm{Cl}_{2}$ and, in addition, the reduced $R_{\mathrm{f}}$ range $(0.02-0.75$ and $0.06-0.80$, respectively) suggests resolution is likely to be poorer. (iii) The DMC and EtOAc data also established excellent distribution with good $R_{\mathrm{f}}$ range coverage (0.04-0.88 and $0.02-0.84$, respectively) and good homogeneity of the data sets (median $R_{\mathrm{f}}=0.55$ and 0.49 , respectively). Overall, based on this analysis it would appear that $\mathrm{MeOH}-\mathrm{CPME}$ offers the highest potential for replacement of $\mathrm{MeOH}-\mathrm{CH}_{2} \mathrm{Cl}_{2}$ in this context, however, $\mathrm{MeOH}-\mathrm{DMC}$ and i-PrOH-EtOAc are also likely to offer considerable utility.

Based on the box plot analysis above, we sought to further analyse our data in order to establish if more in depth correlations existed between the selected replacement solvents and $\mathrm{CH}_{2} \mathrm{Cl}_{2}$. Accordingly, we employed Spotfire ${ }^{10}$ to survey our data for relationships between the data sets for each binary eluent system. This analysis revealed even stronger support for CPME as a candidate for replacement of $\mathrm{CH}_{2} \mathrm{Cl}_{2}$. Fig. 5 shows how the data for $\mathrm{MeOH}-\mathrm{CH}_{2} \mathrm{Cl}_{2}$ correlates with the data for $\mathrm{MeOH}-$ CPME at the $10 \%$ modifier level.

We were pleased to note a strong correlation between the data sets from this analysis with an $R^{2}$ of 0.71 . Similar trends were observed for the $5 \%$ and $20 \%$ modifier data sets $\left(R^{2}=0.55\right.$ and 0.65 , respectively). Interestingly, as shown in Fig. 5 , the majority of outliers were acidic in nature, for example compounds 5, 10, 20, 37, 86, and 91. An additional trend was observed for the acidic compounds where, in general and over the entire data set, these seem to run to higher $R_{\mathrm{f}}$ in $\mathrm{MeOH}-\mathrm{CPME}$ mixtures than in equivalent $\mathrm{MeOH}-\mathrm{CH}_{2} \mathrm{Cl}_{2}$ mixtures. By contrast, certain amine derivatives, for example compounds 66, 79, 93, and 95, exhibited lower $R_{\mathrm{f}}$ values in the $\mathrm{MeOH}-\mathrm{CPME}$ eluent as compared to $\mathrm{MeOH}-\mathrm{CH}_{2} \mathrm{Cl}_{2}$. As predicted from the box plot analysis, similar Spotfire-based mining of the other alternative eluents revealed much poorer correlation with the $\mathrm{MeOH}-\mathrm{CH}_{2} \mathrm{Cl}_{2}$ data and, accordingly, these are less likely to function effectively as direct replacements for $\mathrm{CH}_{2} \mathrm{Cl}_{2}+$ Based on all of the above, we believe that $\mathrm{MeOH}-\mathrm{CPME}$ is a potential viable replacement for $\mathrm{MeOH}-\mathrm{CH}_{2} \mathrm{Cl}_{2}$ within chromatographic purification.

\section{Conclusions}

In summary, we have evaluated several alternative solvents as potential replacements for $\mathrm{CH}_{2} \mathrm{Cl}_{2}$ as the bulk medium for chromatographic purification of a broad range of polar fragments and more complex molecules with functionality frequently encountered within Medicinal Chemistry programmes. Overall, we have established several general trends that may assist in the replacement of $\mathrm{CH}_{2} \mathrm{Cl}_{2}$ in $\mathrm{MeOH}$-based purification processes. Specifically, based on our general analysis of the whole data set, CPME would appear to offer considerable potential as a direct replacement for $\mathrm{CH}_{2} \mathrm{Cl}_{2}$ in binary eluents using $\mathrm{MeOH}$ as the modifier with $\mathrm{MeOH}-\mathrm{DMC}$ and i-PrOH-EtOAc also offering some potential advantages. We have also observed some intriguing trends in relation to certain acidic and basic compounds, which may be useful to laboratory practitioners. Overall, based on the work detailed here and related emerging studies, we believe that replacement of $\mathrm{CH}_{2} \mathrm{Cl}_{2}$ would not only be possible but also practical, straightforward, and highly beneficial for sustainable practice in industry and academia.

\section{Acknowledgements}

We are grateful to GlaxoSmithKline and Sigma Aldrich for the kind donation of consumables. DSM wishes to thank the University of Strathclyde Knowledge Transfer Account for postdoctoral funding. Graham Inglis and Dr Simon MacDonald (GSK) are thanked for helpful discussions.

\section{Notes and references}

1 R. K. Henderson, C. Jiménez-González, D. J. C. Constable, S. R. Alston, G. G. A. Inglis, G. Fisher, J. Sherwood, S. P. Binks and A. D. Curzons, Green Chem., 2011, 13, 854.

2 (a) D. J. C. Constable, C. Jiménez-González and R. K. Henderson, Org. Process Res. Dev., 2007, 11, 133; (b) K. Alfonsi, J. Colberg, P. J. Dunn, T. Fevig, S. Jennings, T. A. Johnson, H. P. Kleine, C. Knight, M. A. Nagy, D. A. Perry and M. Stefaniak, Green Chem., 2008, 10, 31; (c) B. W. Cue and J. Zhang, Green Chem. Lett. Rev., 2009, 2, 193; (d) T. W. J. Cooper, I. B. Campbell and S. J. F. MacDonald, Angew. Chem., Int. Ed., 2010, 49, 8082.

3 J. P. Taygerly, L. M. Miller, A. Yee and E. A. Peterson, Green Chem., 2012, DOI: $10.1039 / \mathrm{c} 2 \mathrm{gc} 36064 \mathrm{k}$.

4 (a) G. S. Cooper, A. S. Bale and P. Schlosser, Toxicological Review of Dichloromethane, US EPA, 2011, http://www.epa.gov/iris/toxreviews/ 0070tr.pdf; (b) http://epa.gov/chemfacts/s_dcm.txt; (c) MSDS available at http://www.sigmaaldrich.com

5 R. Neher, in Thin Layer Chromatography, ed. G. B. Marini-Bettolo, Elsevier, Amsterdam, 1964, p. 77.

6 Compounds 83, 94, and 95 were kindly donated by Professor C. J. Suckling, University of Strathclyde, Glasgow, UK.

7 The KNIME program was used to establish the PCA descriptors. This is freely available online at www.knime.org. For further information, see the ESI.t

8 For information on the use of CPME, see: $(a) \mathrm{K}$. Watanabe, N. Yamagiwa and Y. Torisawa, Org. Process Res. Dev., 2007, 11, 251; (b) http://www. zeon.co.jp/business_e/enterprise/spechemi/spechemi5-13.html

9 A. J. B. Watson, Unpublished results.

$10 \mathrm{http}: / /$ spotfire.tibco.com/ 